\title{
Marital status and survival of patients with kidney cancer
}

\author{
Tingru Miao ${ }^{1}$, Yufeng $\mathrm{Li}^{1}$, Xiaoli Sheng ${ }^{1}$ and Dingguo Yao ${ }^{1}$ \\ ${ }^{1}$ First Affiliated Hospital of Zhejiang Chinese Medical University, Hangzhou, China \\ Correspondence to: Dingguo Yao, email: kejiaoke0579@163.com \\ Keywords: kidney cancer, marital status, SEER database, survival analysis \\ Received: May 02, $2017 \quad$ Accepted: August 06, $2017 \quad$ Published: September 16, 2017 \\ Copyright: Miao et al. This is an open-access article distributed under the terms of the Creative Commons Attribution License 3.0 \\ (CC BY 3.0), which permits unrestricted use, distribution, and reproduction in any medium, provided the original author and source \\ are credited.
}

\section{ABSTRACT}

Background: The relationship between marital status and prognosis of kidney cancer has not been explored in detail. In this study, we aimed to investigate the impact of marital status on survival outcomes in kidney cancer.

Methods: We used the Surveillance, Epidemiology and End Results (SEER) program to identify 112860 patients with kidney cancer diagnosed in 2004 through 2013. Kaplan-Meier methods and multivariable Cox regression models were used to analyze the influence of marital status on overall survival (OS) and cancer-specific survival (CSS).

Results: Married patients had better 5-year OS and CSS compared with patients who were divorced/separated, widowed, and single. After adjusting for known confounders, unmarried patients were at greater risk of overall and cancer-specific mortality, especially the widowed. Moreover, subgroup analysis showed that married still had better prognosis across different SEER stages, ages and sexes.

Conclusions: Our study revealed that marriage is associated with better outcomes of both OS and CSS in kidney cancer patients.

\section{INTRODUCTION}

Marital status has been considered as an independent prognostic factor of survival in several types of cancer [14]. It is well known that social factors may influence the outcome of various diseases [5]. Marital status is regarded as an important social support factor and can greatly affect the patient's emotions, life style, and financial status [6, 7]. Previous studies have shown that being married has a protective role in health and cancer survival compared with being unmarried [8, 9].

Kidney cancer accounts for nearly $2 \%$ of all cancers, and there were an estimated 338,000 newly diagnosed cases and 143,000 deaths due to kidney cancer in 2012 [10]. In addition, $70 \%$ of new cases occurred in developed countries. Incidence and mortality rates of kidney cancer have been increasing in most countries over the past 30 years [11]. It is well-established that cigarette smoking, obesity and hypertension are risk factors for renal cell cancer [12]. However, little is known regarding the association between marital status and survival of patients with kidney cancer. In this study, we used data from the Surveillance, Epidemiology, and End Results (SEER) program to explore the impact of marital status on survival outcomes of kidney cancer patients.

\section{RESULTS}

Patient characteristics

We investigated a total of 112860 kidney cancer patients in the SEER database from 2004 to 2013, including 71549 (63.4\%) male and 41311 (36.6\%) female. Most patients are white $(82.3 \%)$. According to marital status, $71328(63.2 \%)$ were married at diagnosis, and 41352 (36.8\%) were unmarried, including 11896 (10.5\%) divorced/separated, 13160 (11.7\%) widowed, and 16467 $(14.6 \%)$ single. Compared with unmarried patients, the married patients were more likely to be diagnosed at an earlier stage and receive treatment. The widowed 
Table 1: Baseline clinicopathological features of kidney cancer patients in SEER database

\begin{tabular}{|c|c|c|c|c|c|c|}
\hline \multirow{2}{*}{ Characteristic } & Total & Married & Divorced/Separated & Widowed & Single & \multirow{2}{*}{$P$ value } \\
\hline & $112860(100)$ & $71328(63.2)$ & $11896(10.5)$ & $13160(11.7)$ & $16476(14.6)$ & \\
\hline Sex & & & & & & $<0.001$ \\
\hline Male & $71549(63.4)$ & $50344(70.6)$ & $6697(56.3)$ & $3973(30.2)$ & $10535(63.9)$ & \\
\hline Female & 41311(36.6) & 20984(29.4) & $5199(43.7)$ & $9187(69.8)$ & $5941(36.1)$ & \\
\hline Age & & & & & & $<0.001$ \\
\hline$<60$ & $43627(38.7)$ & $27709(38.8)$ & $5307(44.6)$ & $847(6.4)$ & $9764(59.3)$ & \\
\hline $60-70$ & $31801(28.2)$ & $21745(30.5)$ & $3859(32.4)$ & $2347(17.8)$ & $3850(23.4)$ & \\
\hline $70-80$ & 24332(21.6) & $15603(21.9)$ & 2093(17.6) & $4607(35.0)$ & $2029(12.3)$ & \\
\hline$>80$ & 13100(11.6) & $6271(8.8)$ & $637(5.4)$ & $5359(40.7)$ & $833(5.1)$ & \\
\hline Race & & & & & & $<0.001$ \\
\hline White & $92909(82.3)$ & $60549(84.9)$ & $9474(79.6)$ & $10975(83.4)$ & $11911(72.3)$ & \\
\hline Black & 13076(11.6) & $6045(8.5)$ & $1920(16.1)$ & $1535(11.7)$ & $3576(21.7)$ & \\
\hline $\begin{array}{l}\text { American Indian/ } \\
\text { Alaska Native }\end{array}$ & $952(0.8)$ & $470(0.7)$ & $125(1.1)$ & $117(0.9)$ & $240(1.5)$ & \\
\hline Asian/Pacific Islander & $5324(4.7)$ & $3853(5.4)$ & $318(2.7)$ & $505(3.8)$ & $648(3.9)$ & \\
\hline Unknown & $599(0.5)$ & $411(0.6)$ & $59(0.5)$ & $28(0.2)$ & $101(0.6)$ & \\
\hline Grade & & & & & & $<0.001$ \\
\hline High/Moderate & $51438(45.6)$ & $33725(47.3)$ & $5421(45.6)$ & $4745(36.1)$ & $7547(45.8)$ & \\
\hline Poor/Undifferentiation & $29071(25.7)$ & $19216(26.9)$ & $3052(25.7)$ & 2604(19.8) & $4199(25.5)$ & \\
\hline Unknown & $32351(28.7)$ & $18387(25.8)$ & $3423(28.8)$ & $5811(44.2)$ & $4730(28.7)$ & \\
\hline TNM & & & & & & $<0.001$ \\
\hline I & $63996(56.7)$ & $41329(57.9)$ & $6733(56.6)$ & $6552(49.8)$ & 9382(56.9) & \\
\hline II & $9309(8.2)$ & $5943(8.3)$ & $986(8.3)$ & $870(6.6)$ & $1510(9.2)$ & \\
\hline III & $14165(12.6)$ & $9441(13.2)$ & $1358(11.4)$ & 1511(11.5) & $1855(11.3)$ & \\
\hline IV & 17801(15.8) & $10524(14.8)$ & 2044(17.2) & 2552(19.4) & $2681(16.3)$ & \\
\hline Unknown & $7589(6.7)$ & $4091(5.7)$ & $775(6.5)$ & $1675(12.7)$ & $1048(6.4)$ & \\
\hline SEER Stage & & & & & & $<0.001$ \\
\hline Localized & $76192(67.5)$ & $48989(68.7)$ & $8025(67.5)$ & $7877(59.9)$ & $11301(68.6)$ & \\
\hline Regioned & $16162(14.3)$ & $10653(14.9)$ & $1555(13.1)$ & $1810(13.8)$ & 2144(13.0) & \\
\hline Distant & $16786(14.9)$ & 9912(13.9) & 1932(16.2) & 2394(18.2) & $2548(15.5)$ & \\
\hline Unstage & $3720(3.3)$ & $1774(2.5)$ & $384(3.2)$ & $1079(8.2)$ & $483(2.9)$ & \\
\hline Therapy & & & & & & $<0.001$ \\
\hline Surgery, radiation or both & $94075(83.4)$ & $62033(87.0)$ & $9803(82.4)$ & $8581(65.2)$ & $13658(82.9)$ & \\
\hline No surgery, radiation & $17823(15.8)$ & $8845(12.4)$ & $1980(16.6)$ & $4354(33.1)$ & $2644(16.0)$ & \\
\hline Unknown & $962(0.9)$ & $450(0.6)$ & $113(0.9)$ & $225(1.7)$ & $174(1.1)$ & \\
\hline
\end{tabular}


group had the highest percentage of women, the most elderly patients, and the lowest proportion of stage I and localized patients compared with the other groups and more were less likely to receive treatment. The baseline clinicopathological features are shown in Table 1.

\section{Impact of marital status on overall survival in the SEER database}

We performed a Kaplan-Meier analysis to reveal the difference in overall survival (OS) according to marital status (log-rank test $\mathrm{p}<0.001$ ) (Figure 1A). The 5-year OS rate was $69.5 \%$ in the married group, $63.2 \%$ in the divorced/separated group, $46.4 \%$ in the widowed group, and $67.2 \%$ in the single group. In addition to marital status, the results of the Kaplan-Meier analysis indicated that sex, age, race, grade, TNM stage, SEER stage, and therapy were significantly associated with OS in these patients. Cox regression was also used to adjust the variables mentioned above in the multivariate analysis, and marital status was found to be an independent prognostic factor for overall survival (married, reference; divorced/separated, $\mathrm{HR}=1.25,95 \%$ CI 1.21-1.30, $\mathrm{P}<0.001$; widowed, $\mathrm{HR}=1.25,95 \%$ CI 1.21-1.29, $\mathrm{P}<0.001$; single, $\mathrm{HR}=1.18,95 \%$ CI 1.14-1.22, $\mathrm{P}<0.001$ ) (Table 2).

\section{Impact of marital status on cancer-specific survival in the SEER database}

Similarly, the survival curve showed that cancerspecific survival (CSS) among groups with different marital status was significant (log-rank test $\mathrm{P}<0.001$ ) (Figure 1B). The 5-year CSS rates for the married group, the divorced/separated group, the widowed

A

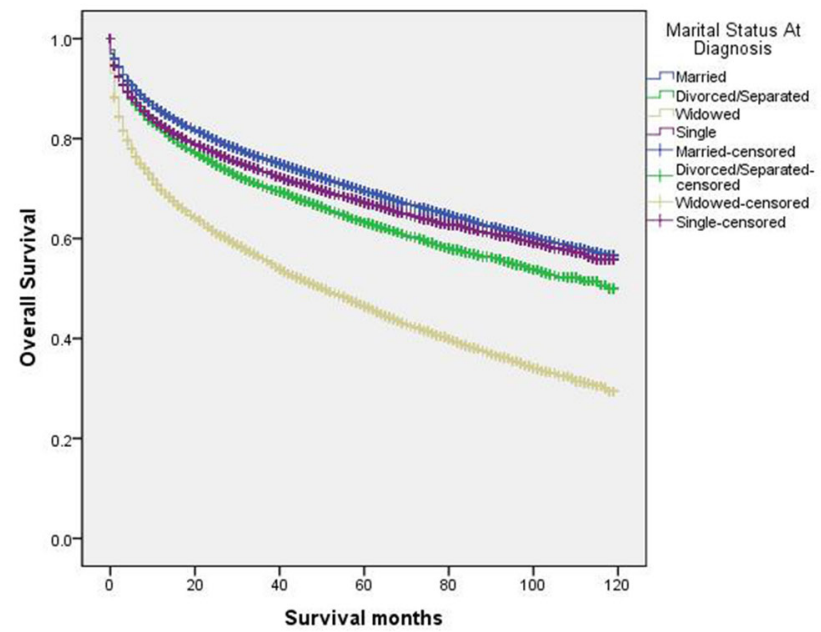

group and the single group were $82.1 \%, 77.8 \%, 70.4 \%$ and $80.1 \%$, respectively. Among clinicopathological variables, sex, age, race, grade, TNM stage, SEER stage, therapy, and marital status were identified as risk factors for predicting CSS based on a KaplanMeier analysis. When a multivariate analysis with Cox regression was performed, marital status was confirmed to be an independent prognostic factor for kidney cancer prognosis (married, reference; divorced/separated, $\mathrm{HR}=1.20$ ，95\% CI 1.15-1.26， $\mathrm{P}<0.001$; widowed, $\mathrm{HR}=1.24,95 \%$ CI 1.19-1.30, $\mathrm{P}<0.001$; single, $\mathrm{HR}=1.14$, 95\% CI 1.09-1.19, $\mathrm{P}<0.001$ ) (Table 3 ). In addition, age ( $\geq 60 \mathrm{y}$ ), poor/undifferentiated, TNM stage, SEER stage, and no surgery and/or radiotherapy were associated with poorer CSS.

\section{Subgroup analysis for evaluating the effect of marital status on CSS}

We further explored the effects of marital status on CSS according to SEER stage (Figure 2 and Table 4). Marital status was still an independent prognostic factor at each stage, both in the univariate and multivariate analysis $(\mathrm{P}<0.001)$. Moreover, we observed some interesting findings. The widowed patients had poorer CSS in localized, regional and distant stages compared with the other groups, and being widowed was associated with a higher risk of mortality compared with being married in the localized stage $(\mathrm{HR}=1.51,95 \% \mathrm{CI} 1.36$ $1.67, \mathrm{P}<0.001)$ and the distant stage $(\mathrm{HR}=1.22,95 \% \mathrm{CI}$ $1.08-1.37, \mathrm{P}<0.001)$. The survival difference between the single and married groups was not apparent in the localized stage $(\mathrm{P}=0.679)$. Furthermore, a previous study showed that prognosis of kidney cancer was associated

B

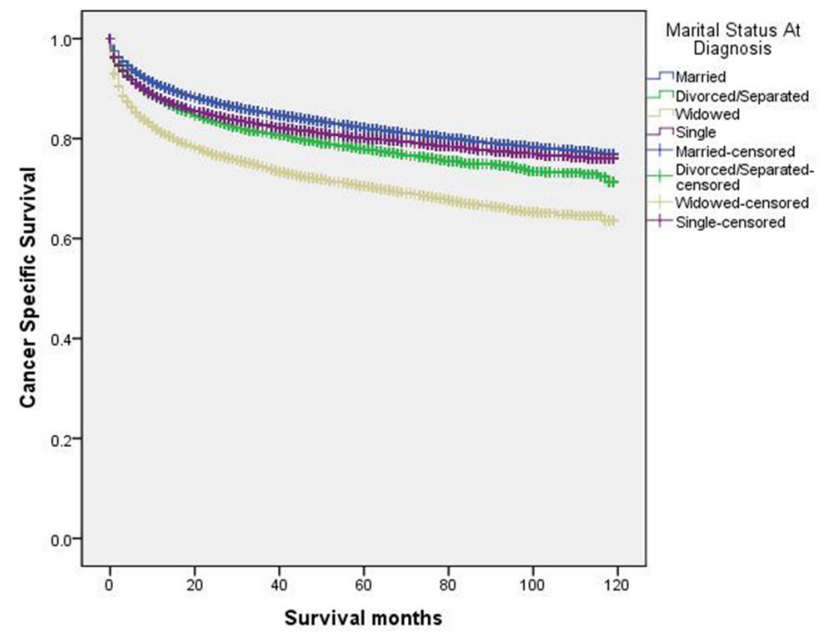

Figure 1: Survival curves in kidney cancer patients according to marital status. (A) Overall survival: $\chi 2=3122.46, \mathrm{P}<0.001$. (B) Cancer specific survival: $\chi 2=1039.78, \mathrm{P}<0.001$. 
Table 2: Univariate and multivariate analysis for overall survival (OS) in kidney cancer patients

\begin{tabular}{|c|c|c|c|c|c|c|}
\hline \multirow{2}{*}{ Variables } & \multirow{2}{*}{$\begin{array}{l}\text { 5-year } \\
\text { OS }\end{array}$} & \multicolumn{2}{|c|}{ Univariate analysis } & \multicolumn{3}{|c|}{ Multivariate analysis } \\
\hline & & $\log \operatorname{rank} X^{2}$ & P value & HR & $95 \% \mathrm{CI}$ & P value \\
\hline \multicolumn{7}{|l|}{ Sex } \\
\hline Male & $64.70 \%$ & 48.23 & $<0.001$ & Reference & & \\
\hline Female & $67.50 \%$ & & & 0.89 & $0.87-0.91$ & $<0.001$ \\
\hline \multicolumn{7}{|l|}{ Age } \\
\hline$<60$ & $77.90 \%$ & 12093.60 & $<0.001$ & Reference & & \\
\hline $60-70$ & $68.70 \%$ & & & 1.38 & $1.34-1.42$ & $<0.001$ \\
\hline $70-80$ & $58.60 \%$ & & & 1.84 & $1.78-1.89$ & $<0.001$ \\
\hline$>80$ & $32.20 \%$ & & & 2.61 & $2.52-2.71$ & $<0.001$ \\
\hline \multicolumn{7}{|l|}{ Race } \\
\hline White & $65.60 \%$ & 136.86 & $<0.001$ & Reference & & \\
\hline Black & $65.00 \%$ & & & 1.07 & $1.04-1.11$ & $<0.001$ \\
\hline $\begin{array}{l}\text { American Indian/ } \\
\text { Alaska Native }\end{array}$ & $63.40 \%$ & & & 0.97 & $0.87-1.09$ & 0.587 \\
\hline Asian/Pacific Islander & $68.50 \%$ & & & 0.87 & $0.82-0.91$ & $<0.001$ \\
\hline Unknown & $90.50 \%$ & & & 0.31 & $0.23-0.42$ & $<0.001$ \\
\hline \multicolumn{7}{|l|}{ Grade } \\
\hline High/Moderate & $83.10 \%$ & 16886.37 & $<0.001$ & Reference & & \\
\hline Poor/Undifferentiation & $60.50 \%$ & & & 1.70 & $1.65-1.75$ & $<0.001$ \\
\hline Unknown & $42.00 \%$ & & & 1.65 & $1.60-1.71$ & $<0.001$ \\
\hline \multicolumn{7}{|l|}{ TNM } \\
\hline I & $83.10 \%$ & 59552.13 & $<0.001$ & Reference & & \\
\hline II & $76.80 \%$ & & & 1.43 & $1.36-1.50$ & $<0.001$ \\
\hline III & $63.10 \%$ & & & 0.83 & $0.75-0.93$ & 0.001 \\
\hline IV & $10.30 \%$ & & & 2.04 & $1.81-2.30$ & $<0.001$ \\
\hline Unknown & $42.90 \%$ & & & 1.54 & $1.44-1.65$ & $<0.001$ \\
\hline \multicolumn{7}{|l|}{ SEER Stage } \\
\hline Localized & $81.60 \%$ & 61354.67 & $<0.001$ & Reference & & \\
\hline Regioned & $58.90 \%$ & & & 2.73 & $2.46-3.03$ & $<0.001$ \\
\hline Distant & $9.40 \%$ & & & 4.27 & $3.79-4.81$ & $<0.001$ \\
\hline Unstage & $25.90 \%$ & & & 1.69 & $1.57-1.83$ & $<0.001$ \\
\hline \multicolumn{7}{|l|}{ Marital Status } \\
\hline Married & $69.50 \%$ & 3122.46 & $<0.001$ & Reference & & \\
\hline Divorced/Separated & $63.20 \%$ & & & 1.25 & $1.21-1.30$ & $<0.001$ \\
\hline Widowed & $46.40 \%$ & & & 1.25 & $1.21-1.29$ & $<0.001$ \\
\hline Single & $67.20 \%$ & & & 1.18 & $1.14-1.22$ & $<0.001$ \\
\hline \multicolumn{7}{|l|}{ Therapy } \\
\hline $\begin{array}{l}\text { Surgery, radiation or } \\
\text { both }\end{array}$ & $75.00 \%$ & 39178.54 & $<0.001$ & Reference & & \\
\hline No surgery, radiation & $17.60 \%$ & & & 2.49 & $2.41-2.56$ & $<0.001$ \\
\hline Unknown & $30.40 \%$ & & & 2.30 & $2.11-2.50$ & $<0.001$ \\
\hline
\end{tabular}


Table 3: Univariate and multivariate analysis for cancer specific survival (CSS) in kidney cancer patients

\begin{tabular}{|c|c|c|c|c|c|c|}
\hline \multirow{2}{*}{ Variables } & \multirow{2}{*}{$\begin{array}{l}\text { 5-year } \\
\text { CSS }\end{array}$} & \multicolumn{2}{|c|}{ Univariate analysis } & \multicolumn{3}{|c|}{ Multivariate analysis } \\
\hline & & Log rank $X^{2}$ & P value & HR & $95 \%$ CI & P value \\
\hline \multicolumn{7}{|l|}{ Sex } \\
\hline Male & $79.50 \%$ & 13.26 & $<0.001$ & Reference & & \\
\hline Female & $81.00 \%$ & & & 1.01 & $0.98-1.04$ & 0.524 \\
\hline \multicolumn{7}{|l|}{ Age } \\
\hline$<60$ & $84.10 \%$ & 2813.62 & $<0.001$ & Reference & & \\
\hline $60-70$ & $81.20 \%$ & & & 1.07 & $1.03-1.11$ & 0.001 \\
\hline $70-80$ & $79.00 \%$ & & & 1.14 & $1.09-1.19$ & $<0.001$ \\
\hline$>80$ & $63.30 \%$ & & & 1.52 & $1.45-1.60$ & $<0.001$ \\
\hline \multicolumn{7}{|l|}{ Race } \\
\hline White & $79.90 \%$ & 79.39 & $<0.001$ & Reference & & \\
\hline Black & $81.60 \%$ & & & 0.99 & $0.95-1.04$ & 0.736 \\
\hline $\begin{array}{l}\text { American Indian/Alaska } \\
\text { Native }\end{array}$ & $75.90 \%$ & & & 1.00 & $0.87-1.16$ & 0.970 \\
\hline Asian/Pacific Islander & $78.90 \%$ & & & 0.98 & $0.92-1.05$ & 0.583 \\
\hline Unknown & $93.60 \%$ & & & 0.42 & $0.29-0.61$ & $<0.001$ \\
\hline \multicolumn{7}{|l|}{ Grade } \\
\hline High/Moderate & $93.90 \%$ & 12360.56 & $<0.001$ & Reference & & \\
\hline Poor/Undifferentiation & $72.30 \%$ & & & 2.22 & $2.12-2.33$ & $<0.001$ \\
\hline Unknown & $62.90 \%$ & & & 2.12 & $2.02-2.23$ & $<0.001$ \\
\hline \multicolumn{7}{|l|}{ TNM } \\
\hline I & $96.20 \%$ & 65843.40 & $<0.001$ & Reference & & \\
\hline II & $87.90 \%$ & & & 3.07 & $2.84-3.32$ & $<0.001$ \\
\hline III & $76.90 \%$ & & & 1.28 & $1.09-1.51$ & 0.003 \\
\hline IV & $17.20 \%$ & & & 3.75 & $3.16-4.46$ & $<0.001$ \\
\hline Unknown & $67.20 \%$ & & & 2.57 & $2.28-2.90$ & $<0.001$ \\
\hline \multicolumn{7}{|l|}{ SEER Stage } \\
\hline Localized & $94.90 \%$ & 67229.90 & $<0.001$ & Reference & & \\
\hline Regioned & $73.20 \%$ & & & 4.55 & $3.91-5.30$ & $<0.001$ \\
\hline Distant & $15.90 \%$ & & & 8.24 & $7.00-9.73$ & $<0.001$ \\
\hline Unstage & $52.80 \%$ & & & 2.77 & $2.44-3.14$ & $<0.001$ \\
\hline \multicolumn{7}{|l|}{ Marital Status } \\
\hline Married & $82.10 \%$ & 1039.78 & $<0.001$ & Reference & & \\
\hline Divorced/Separated & $77.80 \%$ & & & 1.20 & $1.15-1.26$ & $<0.001$ \\
\hline Widowed & $70.40 \%$ & & & 1.24 & $1.19-1.30$ & $<0.001$ \\
\hline Single & $80.10 \%$ & & & 1.14 & $1.09-1.19$ & $<0.001$ \\
\hline \multicolumn{7}{|l|}{ Therapy } \\
\hline Surgery, radiation or both & $86.00 \%$ & 21427.48 & $<0.001$ & Reference & & \\
\hline No surgery, radiation & $42.00 \%$ & & & 2.09 & $2.00-2.18$ & $<0.001$ \\
\hline Unknown & $53.20 \%$ & & & 2.25 & $2.01-2.51$ & $<0.001$ \\
\hline
\end{tabular}


Table 4: Univariate and multivariate analysis for evaluating marital status on CSS according to different SEER stage

\begin{tabular}{|c|c|c|c|c|c|c|}
\hline \multirow{2}{*}{ Variables } & \multirow{2}{*}{ 5-year CSS } & \multicolumn{2}{|c|}{ Univariate analysis } & \multicolumn{3}{|c|}{ Multivariate analysis } \\
\hline & & Log $\operatorname{rank} X^{2}$ & P value & HR & $95 \% \mathrm{CI}$ & P value \\
\hline \multicolumn{7}{|l|}{ Localized } \\
\hline Marital status & & 554.76 & $<0.001$ & & & \\
\hline Married & $95.60 \%$ & & & Reference & & \\
\hline Divorced/Separated & $94.50 \%$ & & & 1.33 & $1.18-1.49$ & $<0.001$ \\
\hline Widowed & $89.50 \%$ & & & 1.51 & $1.36-1.67$ & $<0.001$ \\
\hline Single & $95.60 \%$ & & & 1.15 & $1.03-1.29$ & 0.013 \\
\hline \multicolumn{7}{|l|}{ Regional } \\
\hline Marital status & & 63.77 & $<0.001$ & & & \\
\hline Married & $75.00 \%$ & & & Reference & & \\
\hline Divorced/Separated & $70.50 \%$ & & & 1.11 & $0.99-1.25$ & 0.072 \\
\hline Widowed & $65.70 \%$ & & & 1.06 & $0.95-1.19$ & 0.311 \\
\hline Single & $71.60 \%$ & & & 1.11 & $1.00-1.23$ & 0.050 \\
\hline \multicolumn{7}{|l|}{ Distant } \\
\hline Marital status & & 198.85 & $<0.001$ & & & \\
\hline Married & $17.70 \%$ & & & Reference & & \\
\hline Divorced/Separated & $13.90 \%$ & & & 1.08 & $0.95-1.22$ & 0.271 \\
\hline Widowed & $12.60 \%$ & & & 1.22 & $1.08-1.37$ & 0.001 \\
\hline Single & $13.10 \%$ & & & 1.20 & $1.07-1.35$ & 0.002 \\
\hline
\end{tabular}

with increasing age [13]. Therefore, we further analyzed the survival rates and hazard according to age (Figure 3 and Table 5). Married patients always had better survival in each age group, which were consistent with the above results. However, no survival discrepancy was found among divorced/separated, widowed and single patients. We also performed a further stratified analysis by sex. Unmarried patients were shown to have poorer outcomes both in males and females, which was consistent with the above results (Figure 4 and Table 6).

\section{DISCUSSION}

In this study, we explored the influence of marriage on OS and CSS in kidney cancer patients based on the SEER program. Our study revealed that married patients
A

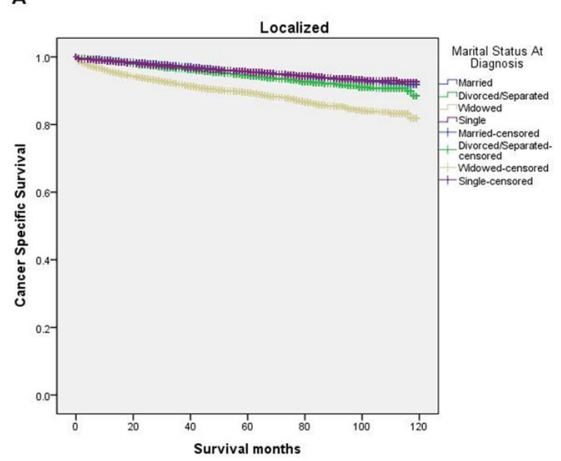

B

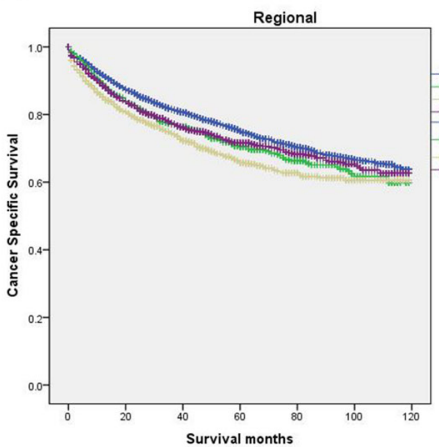

c

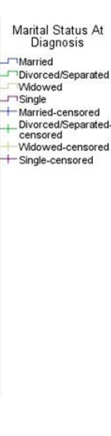

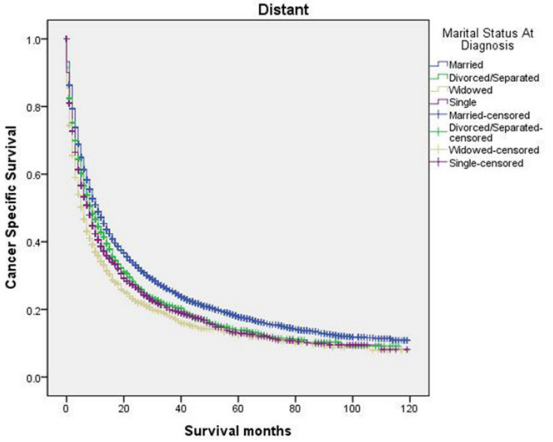

Figure 2: Survival curves in kidney cancer patients on CSS according to marital status in different SEER stages. (A) Localized: $\chi 2=554.76, \mathrm{P}<0.001$; (B) regional: $\chi 2=63.77, \mathrm{P}<0.001$; (C) distant: $\chi 2=198.85, \mathrm{P}<0.001$. 
Table 5: Univariate and multivariate analysis for evaluating marital status on CSS according to different age

\begin{tabular}{|c|c|c|c|c|c|c|}
\hline \multirow{2}{*}{ Variables } & \multirow{2}{*}{ 5-year CSS } & \multicolumn{2}{|c|}{ Univariate analysis } & \multicolumn{3}{|c|}{ Multivariate analysis } \\
\hline & & $\log \operatorname{rank} X^{2}$ & Pvalue & HR & $95 \% \mathrm{CI}$ & P value \\
\hline \multicolumn{7}{|l|}{$<60 \mathrm{y}$} \\
\hline Marital status & & 116.50 & $<0.001$ & & & \\
\hline Married & $85.60 \%$ & & & Reference & & \\
\hline Divorced/Separated & $80.80 \%$ & & & 1.18 & $1.09-1.27$ & $<0.001$ \\
\hline Widowed & $81.80 \%$ & & & 1.22 & $1.03-1.46$ & 0.025 \\
\hline Single & $82.10 \%$ & & & 1.14 & $1.07-1.22$ & $<0.001$ \\
\hline \multicolumn{7}{|l|}{$60-70 y$} \\
\hline Marital status & & 54.83 & $<0.001$ & & & \\
\hline Married & $82.20 \%$ & & & Reference & & \\
\hline Divorced/Separated & $78.30 \%$ & & & 1.17 & $1.08-1.27$ & $<0.001$ \\
\hline Widowed & $79.40 \%$ & & & 1.21 & $1.08-1.34$ & 0.001 \\
\hline Single & $79.80 \%$ & & & 1.13 & $1.03-1.23$ & 0.006 \\
\hline \multicolumn{7}{|l|}{$70-80 y$} \\
\hline Marital status & & 100.93 & $<0.001$ & & & \\
\hline Married & $80.90 \%$ & & & Reference & & \\
\hline Divorced/Separated & $73.90 \%$ & & & 1.23 & $1.11-1.37$ & $<0.001$ \\
\hline Widowed & $75.70 \%$ & & & 1.24 & $1.14-1.35$ & $<0.001$ \\
\hline Single & $77.00 \%$ & & & 1.15 & $1.03-1.28$ & 0.013 \\
\hline \multicolumn{7}{|l|}{$>80 \mathrm{y}$} \\
\hline Marital status & & 101.93 & $<0.001$ & & & \\
\hline Married & $67.70 \%$ & & & Reference & & \\
\hline Divorced/Separated & $59.30 \%$ & & & 1.25 & $1.08-1.45$ & 0.003 \\
\hline Widowed & $58.80 \%$ & & & 1.16 & $1.08-1.26$ & $<0.001$ \\
\hline Single & $62.10 \%$ & & & 1.07 & $0.93-1.23$ & 0.328 \\
\hline
\end{tabular}

had better survival outcomes than unmarried patients, including divorced or separated, widowed, and single patients. In addition, the widowed were at a higher risk of mortality compared with other unmarried groups. Both univariate and multivariate analysis indicated that marital status was an independent prognostic factor for predicting OS and CSS of kidney cancer. Moreover, the survival discrepancy between married and unmarried still existed across different sexes, ages and SEER stages.

The results of our study showed that married patients can enjoy a survival advantage compared with unmarried patients, in agreement with previous studies on other types of cancer [1-4]. However, the mechanisms that drive this correlation are not yet clearly understood. We propose the following underlying mechanisms:
Psychologically, depression is frequently observed in cancer patients [14]. Meta-analyses have shown that the depression in cancer patients can increase cancer mortality by $19 \%$ to $39 \%[15,16]$. A recent study showed that depression was significantly associated with a comparatively higher incidence of hospital admissions, emergency department visits, and health system utilization $[17,18]$. Married patients have a lower risk of psychological distress, anxiety and depression, as their spouses can help share their emotional burden. Compared with married patients, unmarried patients showed not only greater levels of psychological distress but also lower levels of the fighting spirit and higher levels of helplessness [19]. Cancer survivors in the United States reported medication use for anxiety and depression at rates nearly two times those reported by 
the general public, and unmarried are more likely to use medication [20]. In addition, psychological stress may cause poorer adherence to treatment [21].

Physiologically, marriage may have direct influences on cardiovascular, endocrine, immune, neurosensory, and other physiological functions [22]. Endocrine hormones, such as cortisol and catecholamine, can promote tumor growth and metastasis [23]. Married individuals had lower cortisol levels than their never married and previously married counterparts. Cortisol could be regarded as one candidate mechanism accounting for the association of marital status and health [24]. Additionally, the hypothalamus-pituitary-adrenal axis may also have an effect on the immune system, leading to poorer survival $[25,26]$.

Another widely accepted explanation of why married patients have better prognosis of cancer and other diseases is that married patients have better socialeconomic support. It has been reported that married persons are more likely to have better access to medical care and more financial support than unmarried persons [8]. In addition, married couples may have wider health insurance coverage [27]. Thus, spouses might contribute to earlier disease detection [8]. These factors could partly explain why married patients corresponded to a higher percentage of earlier stage cancer in our study. Married patients also have better adherence with prescribed treatments. We found that unmarried, especially widowed, had a lower percentage of surgery and radiotherapy, which could be attributed to the survival difference between the married and unmarried patients. In addition, lifestyles, such as diet habit and behaviors, may also be influenced by marriage [28]. For example, it is well accepted that smoking increases the risk of kidney cancer. Studies show that living without a spouse increases daily smoking rates [29]. Moreover, marriage is associated with an increased probability of cessation for men [30].

Nevertheless, some limitations should be noted. First, the SEER database only provides marital status at the time of diagnosis, which may change after diagnosis. In addition, the quality of the marriage could not be evaluated in the follow-up. SEER also does not record cohabitation status, patients cohabitating with their partners may be categorized as unmarried in the SEER database. Second, we had no direct information on socioeconomic factors, such as educational information, income status, insurance
A

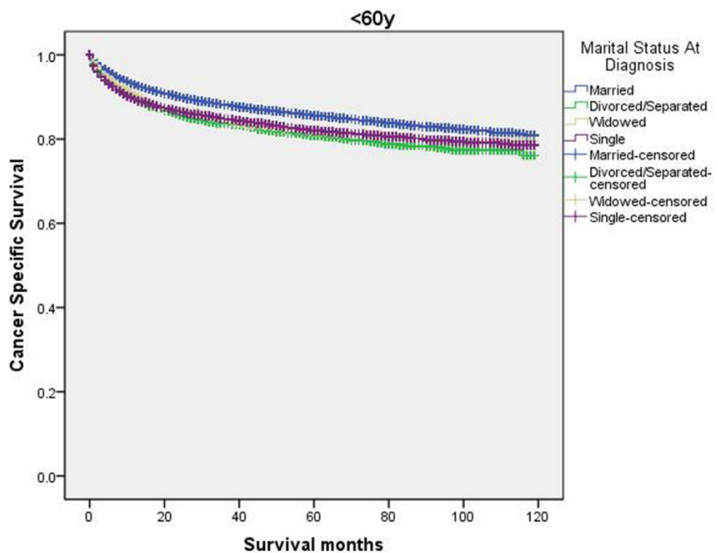

C
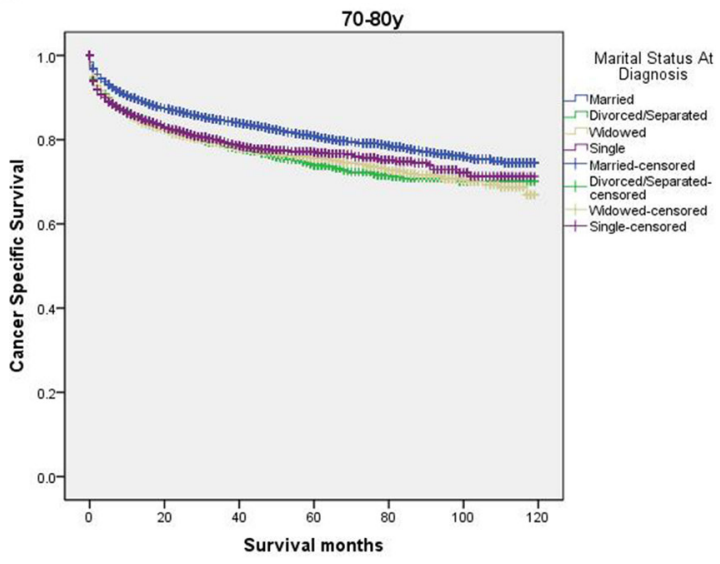

B

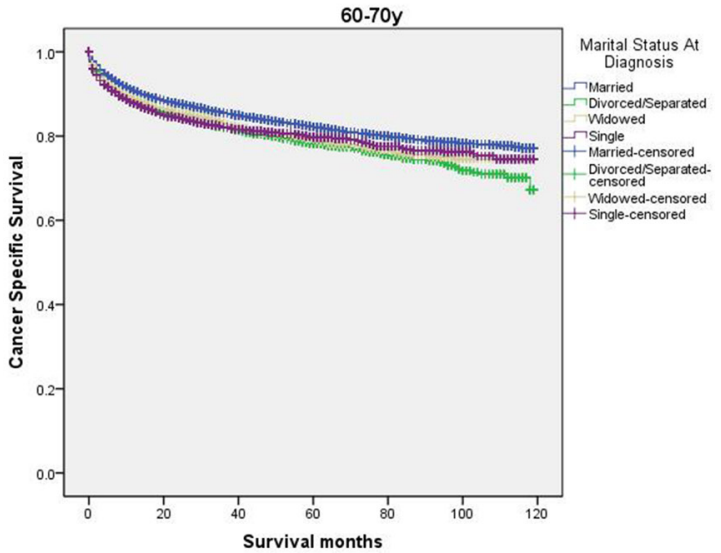

D

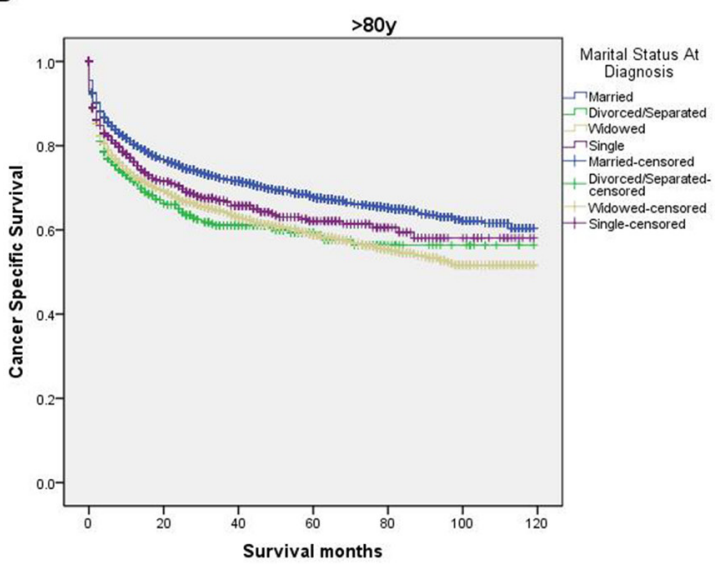

Figure 3: Survival curves in kidney cancer patients on CSS according to marital status in ages. (A) $<60 \mathrm{y}: ~ \chi 2=116.50, \mathrm{P}$ $<0.001$; (B) 60-70y: $\chi 2=54.83, \mathrm{P}<0.001$; (C) 70-80y: $\chi 2=100.93, \mathrm{P}<0.001$ (D) $>80 \mathrm{y}: \chi^{2}=101.93, \mathrm{P}<0.001$. 
Table 6: Univariate and multivariate analysis for evaluating marital status on CSS according to sex

\begin{tabular}{|c|c|c|c|c|c|c|}
\hline \multirow{2}{*}{ Variables } & \multirow{2}{*}{ 5-year CSS } & \multicolumn{2}{|c|}{ Univariate analysis } & \multicolumn{3}{|c|}{ Multivariate analysis } \\
\hline & & Log $\operatorname{rank} X^{2}$ & P value & HR & $95 \% \mathrm{CI}$ & P value \\
\hline \multicolumn{7}{|l|}{ Male } \\
\hline Marital status & & 465.71 & $<0.001$ & & & \\
\hline Married & $81.20 \%$ & & & Reference & & \\
\hline Divorced/Separated & $75.40 \%$ & & & 1.22 & $1.15-1.29$ & $<0.001$ \\
\hline Widowed & $68.60 \%$ & & & 1.28 & $1.20-1.38$ & $<0.001$ \\
\hline Single & $77.90 \%$ & & & 1.17 & $1.11-1.23$ & $<0.001$ \\
\hline \multicolumn{7}{|l|}{ Female } \\
\hline Marital status & & 741.71 & $<0.001$ & & & \\
\hline Married & $84.30 \%$ & & & Reference & & \\
\hline Divorced/Separated & $80.90 \%$ & & & 1.16 & $1.07-1.25$ & $<0.001$ \\
\hline Widowed & $71.20 \%$ & & & 1.14 & $1.07-1.22$ & $<0.001$ \\
\hline Single & $83.80 \%$ & & & 1.05 & $0.97-1.14$ & 0.218 \\
\hline
\end{tabular}

status, and lifestyle (such as smoking and alcohol use), which are not recorded in the SEER database. Third, the SEER database is unable to provide other important survival factors, such as chemotherapy, details of surgery, and other types of therapy. Lastly, as a retrospective study, we could not avoid various forms of bias.

Despite these potential limitations, our study revealed that married kidney cancer patients had survival advantages, while unmarried patients were at higher risk of overall and cancer-specific mortality. We supposed that psychological, physiological and social- economic factors may contribute more to survival outcomes among married patients. Physicians should realize this significant survival difference according to marital status. More social support services and medical interventions should be provided for unmarried patients. Furthermore, future studies should focus on the mechanisms among marital functioning, physiology, and health and on genetic and other variable explanations for marriage-related health outcomes. Additional studies of objective social behavior after changes in marital status are also warranted.
A

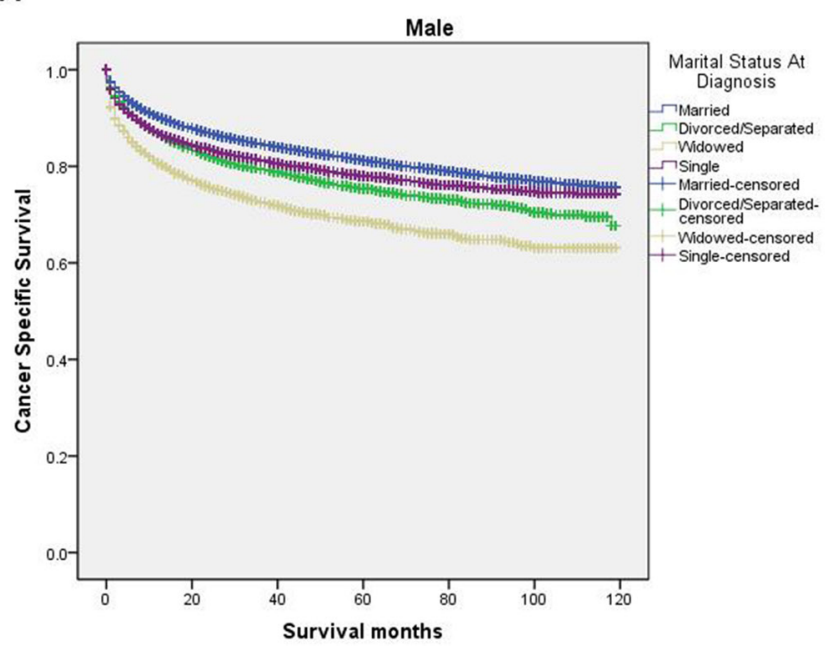

B

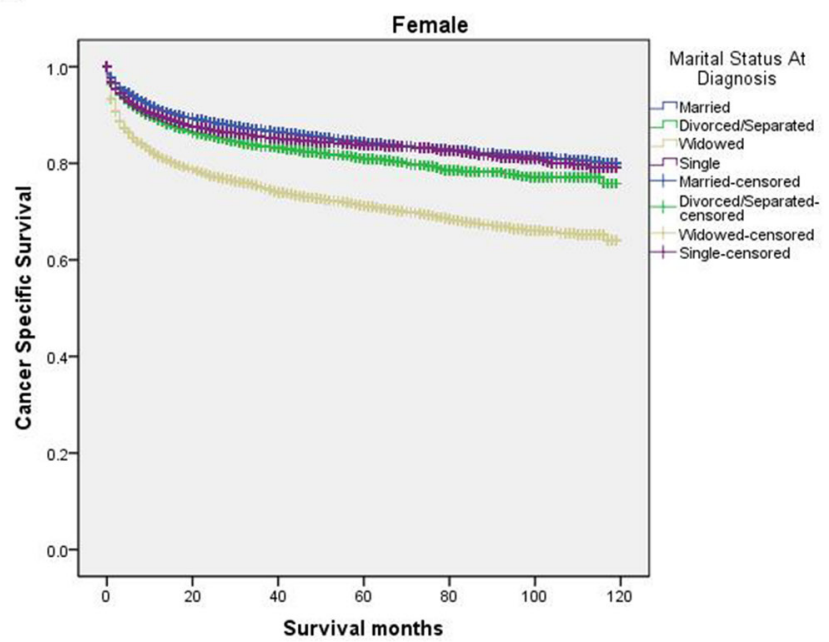

Figure 4: Survival curves in kidney cancer patients on CSS according to marital status in different sexes. (A) Male: $\chi 2$ $=465.71, \mathrm{P}<0.001$; (B) female: $\chi 2=741.71, \mathrm{P}<0.001$. 


\section{MATERIALS AND METHODS}

\section{Data source and patient selection}

This study used data from the SEER database released in March 2017, which covered approximately $28 \%$ of the United States population. By using SEERstat software (version 8.3.4), we searched the database for patients diagnosed between 2004 and 2013 with kidney cancer. Patients aged 18 or older at the time of diagnosis with kidney cancer (International Classification of Diseases for Oncology, Third Edition [ICD-O-3], code C64.9) were included for analysis. Patients were excluded if they had unknown marital status, unknown cause of death or unknown survival time. A total of 112860 patients were included in the cohort. This study was based on public data from the SEER database; we obtained permission to access research data files with the reference number 14920-Nov2015. Because this study did not include the use of human subjects or personal identifying information, this study did not require informed consent.

\section{Study variables}

Variables extracted from the SEER database included marital status, sex, age at diagnosis, race, histological grade, TNM stage, SEER stage, and selection of therapy (surgery/radiotherapy). Marital status is coded as married, divorced or separated, widowed, and single. Age at diagnosis was divided into four groups: $<60$ years, $60-70$ years, $70-80$ years, and $\geq 80$ years. Race was classified as white, black, American Indian/Alaska Native, Asian/Pacific Islander, or unknown. Histological grade was classified as well/moderately differentiated, poorly differentiated/undifferentiated, or unknown. SEER stage was categorized as localized, regional, distant, or unknown. Selection of therapy was divided into three groups: surgery or radiation or both, no surgery or radiation, and unknown.

\section{Outcomes}

The primary outcomes were OS and CSS. OS was calculated from the date of diagnosis to the date of death from any cause. Patients who were alive on the date of last contact or at the follow-up cut-off date were censored. CSS was defined as the time from diagnosis to the date of death due to kidney cancer. Death caused by kidney cancer was considered an event. Patients who died from other causes or who were still alive at the time of the last followup were treated as censored. The follow-up cut-off date was December 31, 2013, according to the SEER database.

\section{Statistical analysis}

The association between marital status and clinicopathological features was assessed by the chi- square $(\chi 2)$ test. Survival curves were generated by the Kaplan-Meier method; differences between the curves were analyzed by using the Log-rank test. Multivariable Cox proportional hazards regression models were used to assess potential risk factors for survival outcomes. All statistical analyses were performed using SPSS for Windows, version 19 (SPSS Inc., Chicago, IL, USA). All $\mathrm{P}$ values were 2 -sided, and $\mathrm{P}<0.05$ was considered statistical significance.

\section{ACKNOWLEDGMENTS}

We thank the Surveillance, Epidemiology, and End Results (SEER) Program tumor registries for providing high quality open resources for researchers. This work was supported by grants from Zhejiang Provincial Natural Science Foundation of China (LY12H07003).

\section{CONFLICTS OF INTEREST}

The authors have no conflicts of interest to declare.

\section{REFERENCES}

1. Li Q, Gan L, Liang L, Li X, Cai S. The influence of marital status on stage at diagnosis and survival of patients with colorectal cancer. Oncotarget. 2015; 6: 7339-47. https://doi. org/10.18632/oncotarget.3129.

2. Shi RL, Qu N, Lu ZW, Liao T, Gao Y, Ji QH. The impact of marital status at diagnosis on cancer survival in patients with differentiated thyroid cancer. Cancer Med. 2016; 5: 2145-54.

3. Li M, Dai CY, Wang YN, Chen T, Wang L, Yang P, Xie D, Mao R, Chen C. Marital status is an independent prognostic factor for tracheal cancer patients: an analysis of the SEER database. Oncotarget. 2016; 7: 77152-62. https://doi. org/10.18632/oncotarget.12809.

4. Aizer AA, Chen MH, McCarthy EP, Mendu ML, Koo S, Wilhite TJ, Graham PL, Choueiri TK, Hoffman KE, Martin NE, Hu JC, Nguyen PL. Marital status and survival in patients with cancer. J Clin Oncol. 2013; 31: 3869-76.

5. Preda A, Voigt K. The social determinants of health: why should we care. Am J Bioeth. 2015; 15: 25-36.

6. Byers TE, Wolf HJ, Bauer KR, Bolick-Aldrich S, Chen VW, Finch JL, Fulton JP, Schymura MJ, Shen T, Van Heest $\mathrm{S}$, Yin X. The impact of socioeconomic status on survival after cancer in the United States: findings from the National Program of Cancer Registries Patterns of Care Study. Cancer. 2008; 113: 582-91.

7. Goodwin JS, Hunt WC, Key CR, Samet JM. The effect of marital status on stage, treatment, and survival of cancer patients. JAMA. 1987; 258: 3125-30.

8. Rendall MS, Weden MM, Favreault MM, Waldron H. The protective effect of marriage for survival: a review and update. Demography. 2011; 48: 481-506. 
9. Kissane DW. Marriage is as protective as chemotherapy in cancer care. J Clin Oncol. 2013; 31: 3852-3.

10. Znaor A, Lortet-Tieulent J, Laversanne M, Jemal A, Bray F. International variations and trends in renal cell carcinoma incidence and mortality. Eur Urol. 2015; 67: 519-30.

11. Chow WH, Dong LM, Devesa SS. Epidemiology and risk factors for kidney cancer. Nat Rev Urol. 2010; 7: 245-57.

12. Capitanio U, Montorsi F. Renal cancer. Lancet. 2017; 387 : 894-906.

13. Hollingsworth JM, Miller DC, Daignault S, Hollenbeck BK. Five-year survival after surgical treatment for kidney cancer: a population-based competing risk analysis. Cancer. 2007; 109: 1763-8.

14. Chochinov HM. Depression in cancer patients. Lancet Oncol. 2001; 2: 499-505.

15. Satin JR, Linden W, Phillips MJ. Depression as a predictor of disease progression and mortality in cancer patients: a meta-analysis. Cancer. 2009; 115: 5349-61.

16. Pinquart $M$, Duberstein PR. Depression and cancer mortality: a meta-analysis. Psychol Med. 2010; 40: 1797-810.

17. Mausbach BT, Irwin SA. Depression and healthcare service utilization in patients with cancer. Psychooncology. 2016; 26: 1133-9. https://doi.org/10.1002/pon.4133.

18. Gunjur A. The added effect of depression on patients with cancer. Lancet Oncol. 2016; 17: e225.

19. Goldzweig G, Andritsch E, Hubert A, Brenner B, Walach N, Perry S, Baider L. Psychological distress among male patients and male spouses: what do oncologists need to know. Ann Oncol. 2010; 21: 877-83.

20. Hawkins NA, Soman A, Buchanan LN, Leadbetter S, Rodriguez JL. Use of medications for treating anxiety and depression in cancer survivors in the United States. J Clin Oncol. 2017; 35: 78-85.

21. Cohen S, Janicki-Deverts D, Miller GE. Psychological stress and disease. JAMA. 2007; 298: 1685-7.

22. Kiecolt-Glaser JK, Newton TL. Marriage and health: his and hers. Psychol Bull. 2001; 127: 472-503.

23. Moreno-Smith M, Lutgendorf SK, Sood AK. Impact of stress on cancer metastasis. Future Oncol. 2010; 6: 1863-81.

24. Chin B, Murphy ML, Janicki-Deverts D, Cohen S. Marital status as a predictor of diurnal salivary cortisol levels and slopes in a community sample of healthy adults. Psychoneuroendocrinology. 2017; 78: 68-75.

25. Reiche EM, Nunes SO, Morimoto HK. Stress, depression, the immune system, and cancer. Lancet Oncol. 2004; 5: 617-25.

26. Dhabhar FS. Effects of stress on immune function: the good, the bad, and the beautiful. Immunol Res. 2014; 58: 193-210.

27. Peters HE, Simon K, Taber JR. Marital disruption and health insurance. Demography. 2014; 51: 1397-421.

28. Umberson D. Gender, marital status and the social control of health behavior. Soc Sci Med. 1992; 34: 907-17.

29. Pennanen M, Broms U, Korhonen T, Haukkala A, Partonen T, Tuulio-Henriksson A, Laatikainen T, Patja K, Kaprio J. Smoking, nicotine dependence and nicotine intake by socioeconomic status and marital status. Addict Behav. 2014; 39: 1145-51.

30. Broms U, Silventoinen K, Lahelma E, Koskenvuo M, Kaprio J. Smoking cessation by socioeconomic status and marital status: the contribution of smoking behavior and family background. Nicotine Tob Res. 2004; 6: 447-55. 